\title{
Interim Clinical Outcomes in Nanocomposite Bone Material Repairing Large Proximal Femoral Defect of Fibrous Dysplasia
}

\author{
Yun Lang, ${ }^{1}$ Ze-ping Yu, ${ }^{1}$ Yan Xiong, ${ }^{1}$ Chong-qi Tu, ${ }^{1}$ Cheng Ren, ${ }^{1}$ Bin Zhang, \\ Hong-sheng Yang, ${ }^{1}$ Fang Yuan, ${ }^{2}$ Hong Li, ${ }^{3}$ Yong-gang Yan, ${ }^{3}$ and Hong Duan ${ }^{1}$ \\ ${ }^{1}$ Deparment of Orthopedics, West China Hospital, Sichuan University, No. 37 Guo Xue Lane, Chengdu, Sichuan 610064, China \\ ${ }^{2}$ Department of Radiology, West China Hospital, Sichuan University, Chengdu, China \\ ${ }^{3}$ School of Physical Science and Technology, Sichuan University, Chengdu, China \\ Correspondence should be addressed to Chong-qi Tu; tuchongqi@163.com and Hong Duan; duanhong1970@126.com
}

Received 14 April 2015; Revised 23 June 2015; Accepted 29 June 2015

Academic Editor: Faik Oktar

Copyright (C) 2015 Yun Lang et al. This is an open access article distributed under the Creative Commons Attribution License, which permits unrestricted use, distribution, and reproduction in any medium, provided the original work is properly cited.

\begin{abstract}
Background and Objectives. To evaluate the clinical effectiveness and safety of using nanocomposite bone material in the repair of large proximal femoral defects that are due to fibrous dysplasia. Method. Thirty-one patients were analyzed retrospectively, including 13 males and 18 females, and the mean age was 30.9 years (13-59). The median follow-up period was 50 months (30-78) and the masses of artificial bone transplants were in the range of $15 \sim 40 \mathrm{~g}$ (average of $23.4 \mathrm{~g}$ ). Functional and radiographic outcomes were evaluated. Results. All wounds healed to grade A. There were no infections, nonspecific inflammatory reactions, rejection reactions, or fractures. One case had fat liquefaction and healed after dressing. All patients had no recurrence until the last follow-up. At the last follow-up, the mean Musculoskeletal Tumor Society's (MSTS) 93 score was $28.42 \pm 1.31$, the mean Harris hip score was 84.23 \pm 8.97 , and mean radiopaque density ratio was $0.78 \pm 0.09$. Radiologic analysis indicated that nanocomposite bone material had been completely incorporated with the host bone within a year. Conclusions. This study indicated that the nanocomposite bone material had biological safety and good biocompatibility. In conclusion, the nanocomposite bone material is an ideal artificial bone substitute and worthy of promotion in the field of orthopedics.
\end{abstract}

\section{Introduction}

Bones are an important organ in our bodies and provide us the freedom to do the things that we want to do. Bones help us to stand up straight, walk, and jump. Many health problems, such as trauma, infection, and tumors, can cause broken bones [1]. Bone tissue can repair itself for small defects, and sometimes cannot heal large bone defects. The treatment of large bone defects represents a considerable challenge in clinical practice $[2,3]$. This situation necessitates the use of bone grafts, including autografts, allografts, and bone substitutes for healing [4]. Autografts have long been acknowledged as the gold standard for bone grafts due to their outstanding osteoinductivity, osteoconductivity, and osteogenicity. However, the following limitations restrict their extensive clinical application: limited supply, donor site injury, and potential risks of infection. Allografts, providing scaffolds for graft sites, are relatively abundant in supply, are osteoconductive, and have been used successfully in bone grafting procedures. However, allografts have their limitations as well, including a slowly creeping substitution process compared to autografts, high risks of disunion or delayed union, and potential risk of disease transmission and antigenicity that induces host rejections. Bone graft substitutes include organic materials, inorganic materials, and composites. Surgeons encounter a serious challenge in searching for the ideal artificial bone substitute for the repair of large bone defects. Biomaterials, due to their comparability with human bone structure, have been demonstrated to be popular in orthopedic surgery [4].

Fibrous dysplasia (FD) of bone is a rare spontaneous benign skeletal disorder characterized by a focal proliferation of fibrous tissue in the bone marrow that manifests in childhood or early adult life and can affect one bone (monostotic), or multiple bones (polyostotic) [5]. According to Ippolito and colleagues [6], in monostotic FD, the most commonly appearing site is on the femur. Clinical symptoms 
TABLE 1: Surgical approach of 31 patients.

\begin{tabular}{|c|c|c|c|}
\hline Lesion type & Proximal femur & Femoral neck and proximal femur & Total \\
\hline Bone material grafting & 7 & 4 & 11 \\
\hline Bone material grafting + internal fixation & 8 & 9 & 17 \\
\hline Bone material + allograft cortical bone plate grafting + internal fixation & 2 & 1 & 3 \\
\hline Total & 17 & 14 & 31 \\
\hline
\end{tabular}

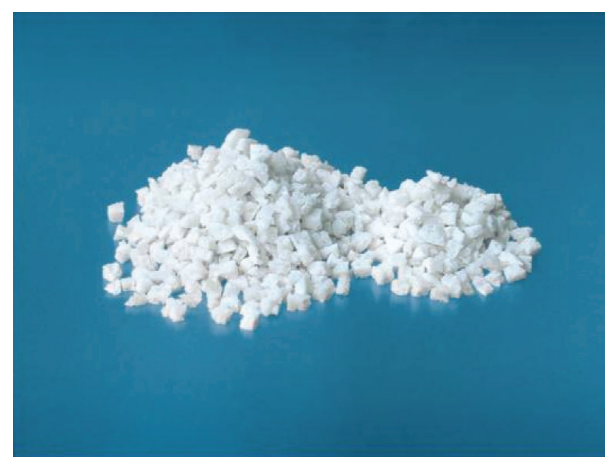

(a)



(b)

FIGURE 1: The morphology of nanocomposite bone material. Notes: (a) nanocomposite bone material with a size of $4 \mathrm{~mm} \times 2 \mathrm{~mm} \times 2 \mathrm{~mm}$; (b) SEM image ( $\times 3,000$ magnification) of nanocomposite bone material with a porosity of $75 \%-85 \%$.SEM: scanning electron microscope.

of FD include bone pain, bone deformities, and pathological fractures [7]. It is well-known that the surgical management of fibrous dysplasia includes intralesional curettage, correction of the deformity, bone grafting, and rigid internal fixation. Various types of surgical treatment are reported, ranging from lesion curettage after bone grafting to massive cortical bone grafting, particularly in lesions of the femoral neck and for intramedullary fixation in extended lesions with deformations [8-10]. The multiple treatment modalities make FD difficult to manage, especially in the proximal femur.

Nanocomposite bone material resembles human bone in terms of structure and mechanical strength, which is useful as a bone repairing material because it has biocompatibility, bioactivity, and good biomechanical properties as confirmed in various research [11-17].

We reviewed 31 patients and reported on our experience in applying nanocomposite bone material (the porous nHA/PA66 composite) to repair large proximal femoral defects of FD.

\section{Materials and Methods}

2.1. Material Introduction. Nanocomposite bone material, which was provided by the Sichuan National Nanotechnology Co., Ltd. (Chengdu, People's Republic of China) and met biological safety standards according to the Chinese GB/T16886 and GB/T16175, was the biomaterial composite and consisted of nanohydroxyapatite (n-HA) and polyamide 66 (PA66) (Figure 1). The diameters of n-HA nanoparticles range from $80 \mathrm{~nm}$ to $100 \mathrm{~nm}$ and the molecular weight is 1,000 , while PA66 has a molecular weight range from 18,000 to 20,000. The mass ratios of n-HA and PA66 in the composite were 35 to 40 . The composite pore size, porosity ratio, and compressive strength were 300 to $700 \mu \mathrm{m}, 75 \%$ to $85 \%$, and 2.0 to $10.0 \mathrm{mPa}$, respectively. For the nanocomposite bone material, it was demonstrated that there was no cytotoxicity or pyrogen. Additionally, the material's hemolysis ratio was $0.59 \%$ [17]. Early animal implantation studies showed that a rapid developing process of bone repair was observed in the nanocomposite, the primer mineralization started at the implant near to the neighboring bone tissue, and the new bone started increasing at 4 weeks. After 12 weeks, new bone formed in the pores, progressively linked with each other, and directly connected with materials. After 24 weeks, the new bone gradually reached toward the center of the implant and exhibited good osteoinductivity and osteocompatibility (Figures 2 and 3). The treatments were undertaken with the understanding and written consent of each subject according to the World Medical Association Declaration of Helsinki (version 2008). The study has been independently reviewed and approved by the Ethics committee at West China Hospital of Sichuan University.

2.2. Clinical Data. From December 2007 to October 2011, there were 31 consecutive patients who fulfilled the following inclusion criteria: (1) the diagnosis of FD should be confirmed by clinical feature, imaging data and pathology; (2) lesions should undergo intralesional curettage, biopsy, nanocomposite bone material transplantation, or rigid internal fixation and allograft used according to the type of lesion; (3) there was complete follow-up data. Exclusion criteria were as follows: (1) the patient showed recurrence; (2) patients contraindicated to operation. There were 13 males and 18 females and the mean age was 30.9 years (13-59). The lesion locations are listed in Table 1 . The size of the lesions ranged from $6.0 \mathrm{~cm} \times 2.5 \mathrm{~cm} \times 2.5 \mathrm{~cm}$ to $10.0 \mathrm{~cm} \times 3.5 \mathrm{~cm} \times 3.5 \mathrm{~cm}$. 


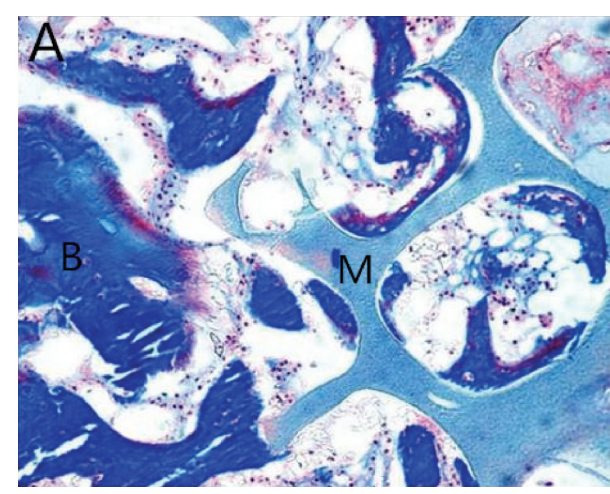

(a)

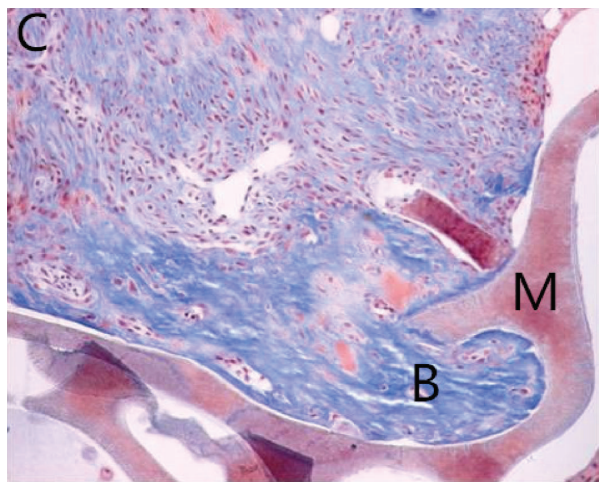

(c)

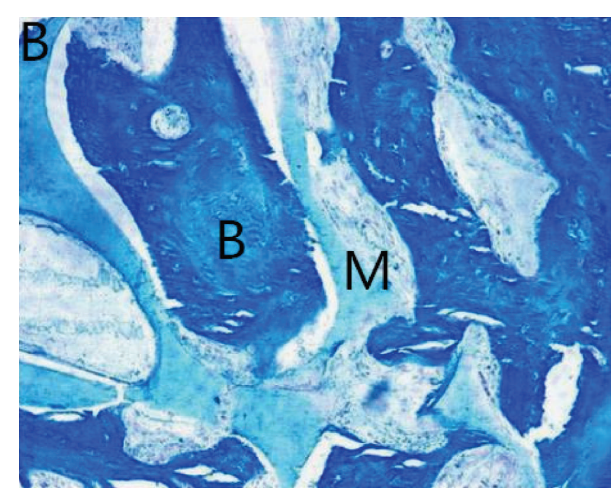

(b)

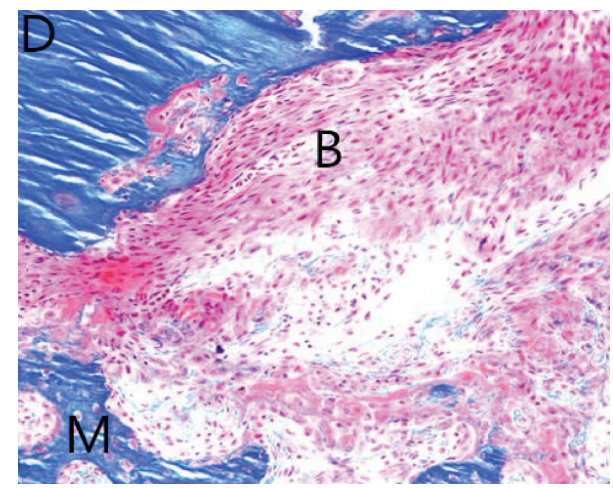

(d)

FIGURE 2: Figure showing how the amount of new bone and calcium crystals increased over time. Note: bone and calcium crystals at (a) 2 weeks, (b) 4 weeks, (c) 12 weeks, and (d) 24 weeks. Masson staining was used, $\times 200$ magnification. After 24 weeks, interconnected porosity was filled completely with new bone tissue. M: material; B: bone.

The average of the implant amount of nanocomposite bone material was $23.4 \mathrm{~g}(15-40)$. We used a software program (Syngo version V35; Siemens Medical Systems, Erlangen, Germany) for quantitative analysis to measure the X-ray radiopaque density value. The value was measured in the grafting section and nonlesion bone area near the operation section, and the grafting-to-nonlesion count ratio ( $\mathrm{G} / \mathrm{N}$ ratio) was then calculated. The normal value of the $\mathrm{G} / \mathrm{N}$ ratio was 1.00 [14]. Both preoperatively and postoperatively, all patients underwent blood investigations, biochemical tests, immune function tests, and hemagglutination inspections.

2.3. Surgical Methods. This procedure included four following steps: orderly intralesional curettage, biopsy, inactivated, and filled with nanocomposite bone material. If the cortical bone was seriously affected, the combination application of allogeneic cortex bone plate and nanocomposite bone material grafting was recommended. The femur reconstruction nail or proximal femoral nail antirotation (PFNA) would be planted for those with a deformity, pathologic fracture, or obviously weakened bone strength. Additionally, the deformity cases underwent valgus osteotomy prior to these steps. The surgery procedure option is shown in Table 1 , which all patients underwent. 22 patients with hip and/or the femoral varus deformity underwent a single level wedgeshaped valgus osteotomy at subtrochanteric region to ensure a neck-shaft angle of $120^{\circ}$; if the deformity combined the femur neck and the subtrochanteric region, double-level valgus osteotomy was performed with the first level at the subtrochanteric region and the second around the dome of the varus femur deformity. Hence, to ensure the healing of the osteotomy site, the second osteotomy site was determined by the host bone quality, with our goal of double-level osteotomy being to restore the neck-shaft angle more than $90^{\circ}$ and rebuild the femur alignment. Three cases with lesions involving a bone cross-section over $50 \%$ and cortical bone defects over $5 \mathrm{~cm}$ underwent double-level osteotomy. Meanwhile, allogeneic cortex bone plate grafting and femur reconstruction nail fixation were performed.

2.4. Postoperative Treatment and Follow-Up. Postoperatively, all patients accepted intravenous infusion cefathiamidine to prevent infection, early isotope exercise of quadriceps, and passive exercise of hips and knees. The clinical evaluation included wound healing, blood investigations, biochemical tests, immune function tests, and hemagglutination inspections one, 2, 3, 6, 9, and 12 months and then every six months after surgery. At the same time, patients were followed up by panoramic X-ray and computed tomography (CT) plain scan, and spiral CT $3 \mathrm{D}$ reconstruction was performed to observe the healing of grafted bone and calculate the $\mathrm{G} / \mathrm{N}$ ratio (image measurement data and statistical data using a double-blind 


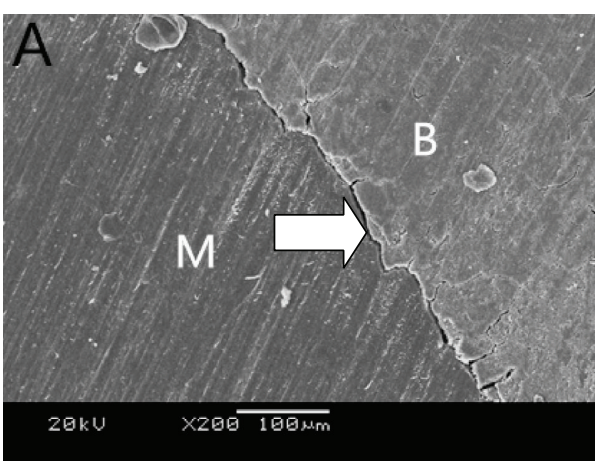

(a)

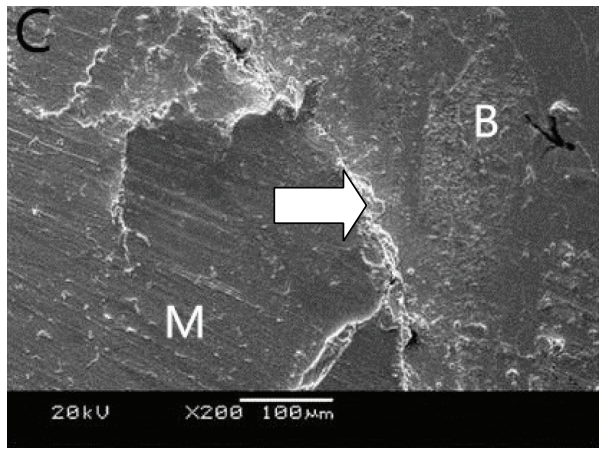

(c)

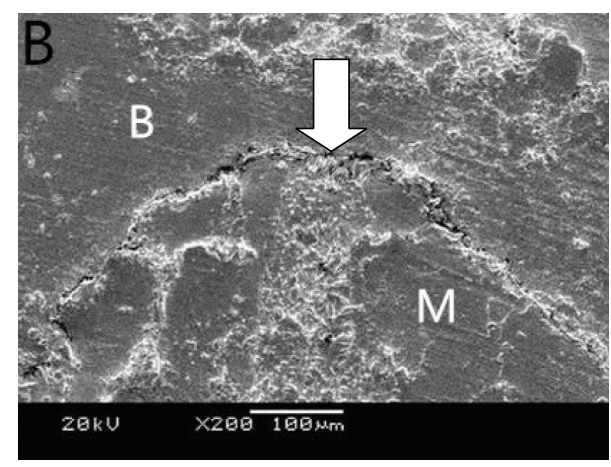

(b)

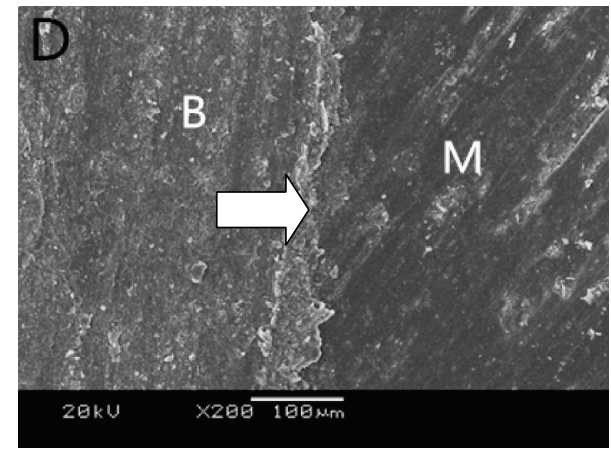

(d)

FIgURE 3: The SEM images $(\times 2,000$ magnification $)$ of the nanocomposite bone material with implantation into the rabbit tibia. Note: the interface of material and bone at (a) 2 weeks, (b) 4 weeks, (c) 12 weeks, and (d) 24 weeks. After 24 weeks, the nanocomposite bone material integrated with host bone completely. SEM: scanning electron microscope; M: material; B: bone.

evaluation). The Harris hip score system [18] and MSTS 93 scoring system [19] were used to analyze limb function.

\subsection{Statistical Methods. Data were coded and analyzed using} SPSS 17.0 (IBM Corporation, Armonk, NY, USA) software. The values are presented as the mean \pm standard deviation (SD). Paired sample $t$-test was used to compare the difference in Harris hip score, MSTS 93 score, and G/N ratio between preoperative and postoperative. One-way analysis of variance (ANOVA) was used to determine differences between the radiopaque density ratios for dependent variables measured at the normal level (age, sex, and bone grafted mass). A value of $P<0.05$ was considered to be statistically significant.

\section{Results}

The median follow-up period was 50 months (30-78). After surgery, all patients had a good general condition. One case had fat liquefaction of their postsurgical wound and was healed by meticulous dressing. The other wounds healed to grade A. All of the patients' blood investigations, biochemical tests, immune function tests, and hemagglutination inspections showed no obvious abnormalities from preoperative to postoperative. No nonspecific inflammation, rejection, recurrence, pathologic fractures, internal fixation loosening, local calcification, or heterotopic ossification were encountered. The grafting site did not show signs of osteoporosis. One case with a severe deformity with increscent femoral neck anteversion angle and proximal femur axis rotation underwent osteotomy. Unfortunately, the dysfunction showed no obvious improvement. This patient accepted another surgery to cut off their adductor, and the function level has gradually improved. Radiographs showed radiolucent zones between the implanted nanocomposite bone material and the neighboring bone tissues. Periodic assessments showed that $25 \%$ of grafting zones were replaced by new bones in 2 to 3 months; after 6 to 9 months, new bone formation rose to $50 \%$. The defects were fully occupied by new bone within one year and reached a radiographically observable bony union (Figures 4 and 5). The $\mathrm{G} / \mathrm{N}$ ratio reflected the differences between grafting and nonlesion areas. New bone grafting area blocked $\mathrm{X}$-ray penetration, and as time went on more new bone grew, the $\mathrm{G} / \mathrm{N}$ ratio decreased, and after one year this ratio entered into the stationary phase. $\mathrm{G} / \mathrm{N}$ ratio was $0.78 \pm 0.09$ one year after surgery (Table 2). There was no significant relationship between age, sex, bone graft mass, and radiopaque density ratio $(P>0.05)$ (Table 3$)$. There was no significant difference in $\mathrm{G} / \mathrm{N}$ ratio between allograft users and nanocomposite bone material isolate users. The Harris hip score was 84.23 \pm 8.97 , and the MSTS score was $28.42 \pm 1.31$ (Table 2).

\section{Discussion}

The management of FD in the proximal femur is a tough challenge in the musculoskeletal tumor surgery field. FD 


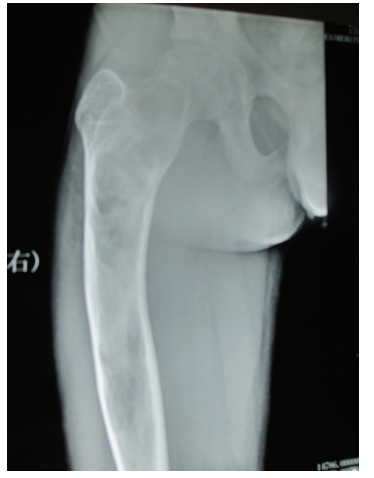

(a)

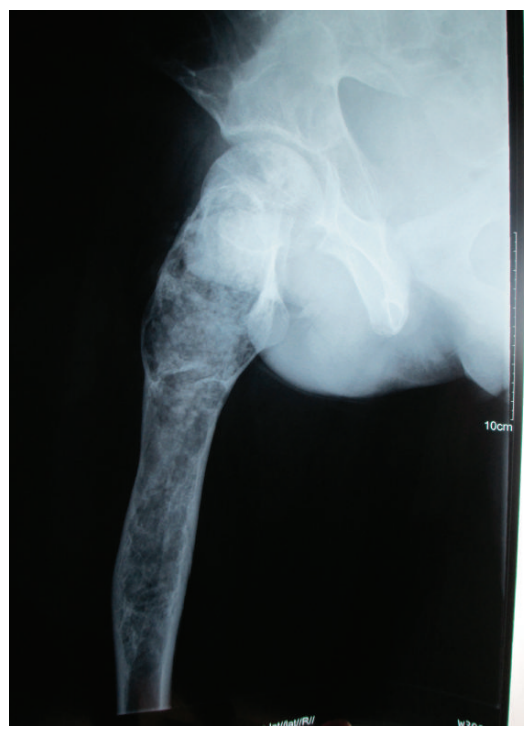

(d)

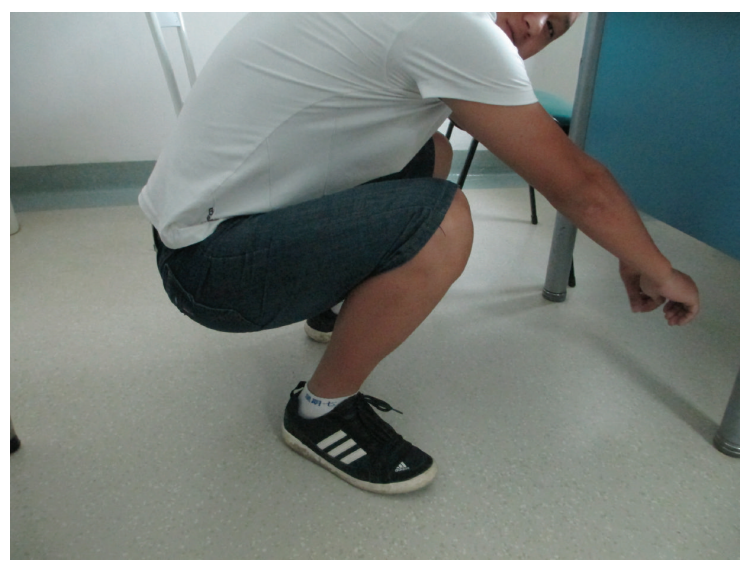

(f)

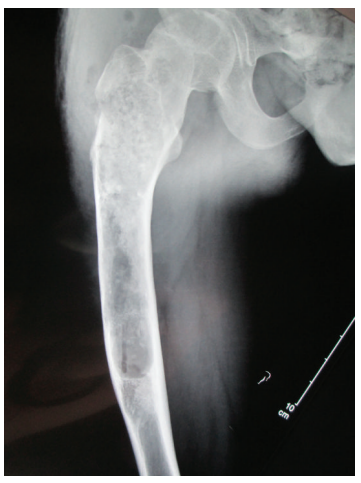

(b)



(c)

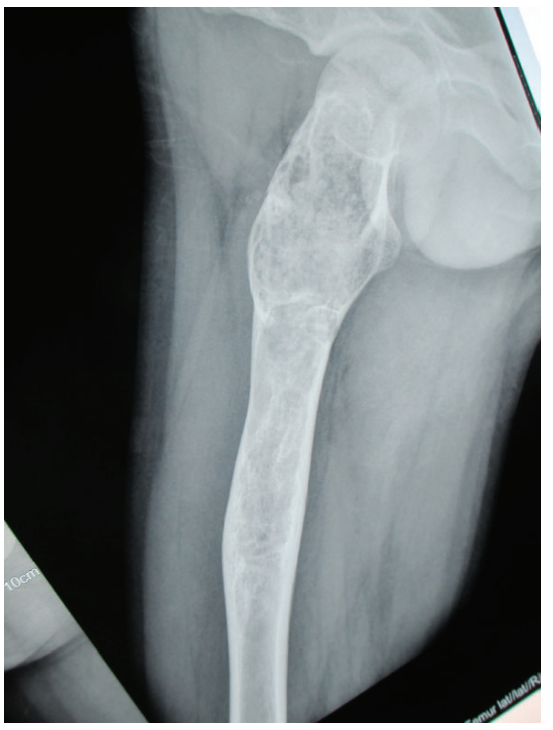

(e)

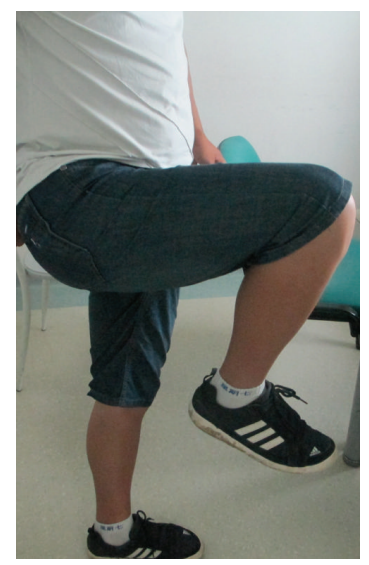

(g)

FIGURE 4: A man, 20 years old, suffered from FD of right proximal femur (a). The patient underwent lesion biopsy, curettage, and nanocomposite bone material grafting. Three months after surgery (b); 6 months after surgery (c); one year after surgery (d); and 3 years follow-up (e). (d) and (e) showed that nanocomposite bone material had been completely incorporated with the host bone, and grafting-tononlesion count ratio was 0.84 . The Harris score was 92 and MSTS score was 29 (f, g). The patient was satisfied by the function. 


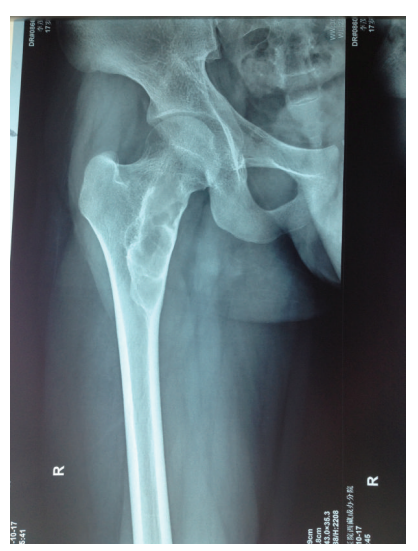

(a1)

(a)

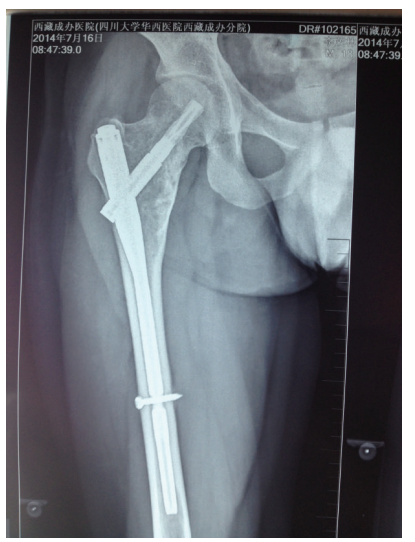

(c1)

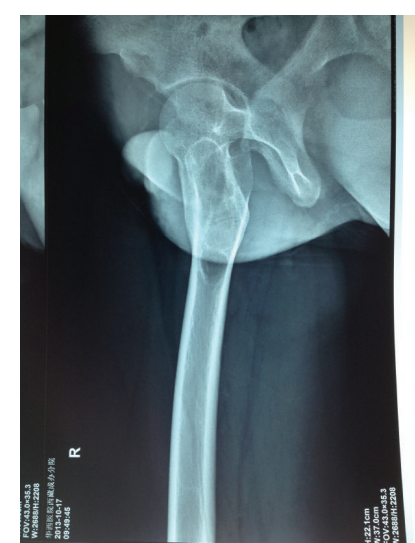

(a2)

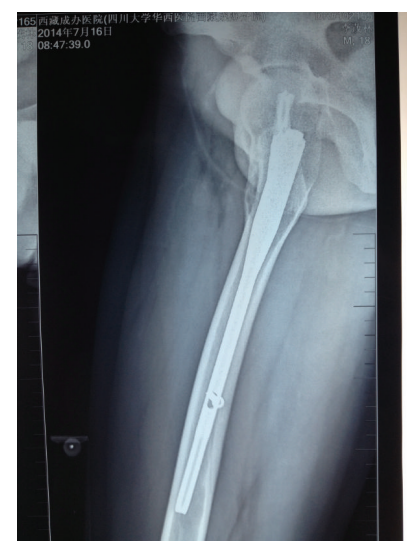

(c2)

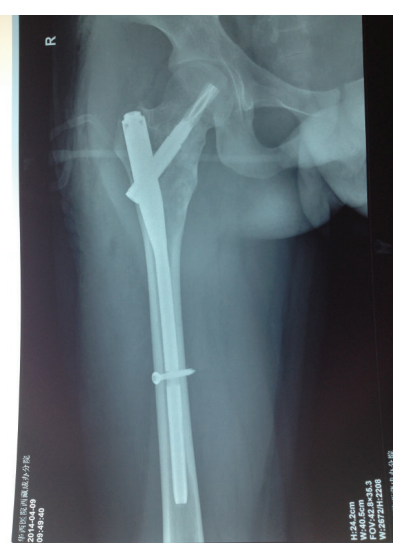

(b1)



(d1)

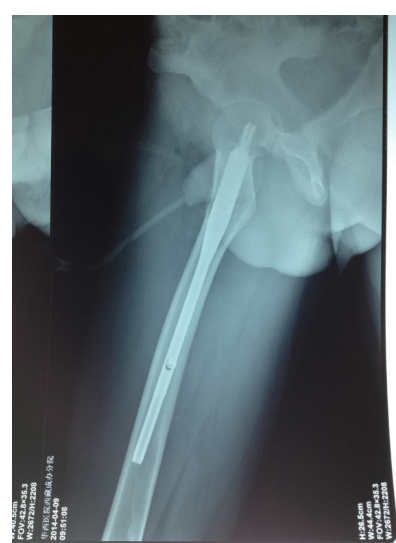

(b2)

(b)

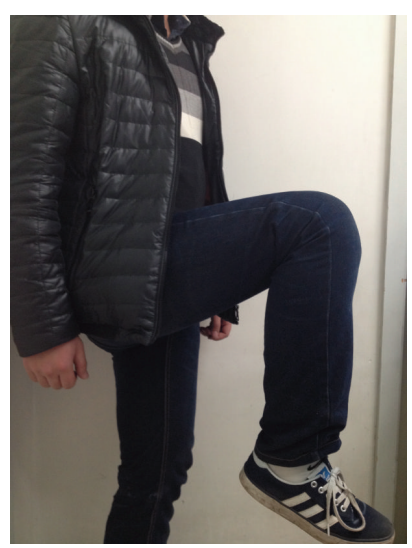

(d2)

(c)

(d)

Figure 5: A man, 22 years old, suffered from FD of right proximal femur (a1, a2). The patient underwent lesion biopsy, intralesion curettage, nanocomposite bone material grafting, and PFNA intramedullary nail fixation. One year (b1, b2) and 3 years (c1, c2). (c1) and (c2) showed that the nanocomposite bone material had been completely incorporated with the host bone, and the fixation was stable. There was no lesion recurrence. The grafting-to-nonlesion count ratio was 0.80 . The Harris score was 93 and MSTS score was 29 (d1, d2). The patient could participate in long distance running.

TABLE 2: Comparison of Harris score, MSTS score, and G/N ratio between preoperative and 1 year follow-up.

\begin{tabular}{lcc}
\hline Items & Before operation & A year after operation \\
\hline Harris score & $62.06 \pm 10.34$ & $84.23 \pm 8.97^{*}$ \\
MSTS score & $23.65 \pm 1.47$ & $28.42 \pm 1.31^{*}$ \\
G/N ratio & $0.49 \pm 0.06$ & $0.78 \pm 0.09^{*}$ \\
\hline
\end{tabular}

${ }^{*}$ Comparison with preoperative evaluation $P<0.01$.

generally weakens biomechanical strength and destroys the anatomic structure of the femur. With the progression of FD, long-term weight bearing, and the traction of hip muscles can cause hip pain, pathological fractures, and varus deformities, which fundamentally affect limb function and can even lead to a high rate of disability $[6,10,20,21]$. Therefore, it is highly important to have early detection and treatment of FD. The surgical management procedure for FD should include the following steps $[6,9,21]$ : (1) correcting the deformity; (2)
TABle 3: Statistical data on 31 patients.

\begin{tabular}{lcccc}
\hline Variable & Total $(N=31)$ & G/N ratio & $F$ & $P$ \\
\hline $\begin{array}{l}\text { Age }^{1} \\
\text { Sex }^{2}\end{array}$ & 31 & $0.78 \pm 0.09$ & 2.403 & 0.088 \\
$\quad$ Female & 18 & $0.80 \pm 0.06$ & & \\
$\quad$ Male & 13 & $0.77 \pm 0.12$ & & \\
$\quad \begin{array}{l}\text { Bone grafted mass } \\
\quad 2\end{array}$ & & & & \\
$\quad<20 \mathrm{~g}$ & 5 & $0.71 \pm 0.20$ & 1.265 & 0.309 \\
$\quad 220 \mathrm{~g}$ & 26 & $0.80 \pm 0.05$ & & \\
\hline
\end{tabular}

$\mathrm{G} / \mathrm{N}$ ratio, grafting-to-nonlesion ratio.

${ }^{1}$ Correlation between the two variables was analyzed by linear correlation.

${ }^{2}$ One-way analysis of variance (ANOVA).

using intralesional curettage, high-speed burring to remove the lesions; (3) bone grafting; (4) rigid internal fixation.

Using intralesional curettage and bone grafting are the principle methods for treating large proximal femoral defects of FD, which can effectively prevent lesion progression, 
rebuild anatomic structures, and heighten mechanical properties of the lesion sites. As previously described, considering the limitations of autografts and allografts, scientists continue to explore new artificial bone replacement products, such as nanocomposite bone material in our institution $[17,22]$.

As a significant component of nanocomposite bone material, n-HA is similar to natural bone in composition and morphology [22]. The early period animal research and in vitro experimental studies demonstrated that the n-HA was biologically safe and had good histocompatibility and its hemolysis ratio was given as $0.59 \%$ without cytotoxicity, allergenicity, or pyrogen reaction $[12,16,17$, 23-26]. Nanocomposite bone material made up for the deficiencies of autografts and allografts to some extent [27, 28]. Moreover, nanocomposite bone material ensured an adequate supply and was convenient for application without additional damage to patients. Apart from bone autografts and allografts, nanocomposite bone material simplified bonegrafting procedures and shortened operation time. Our investigation determined that the 31 patients responded that their function recovered satisfactorily, and their limb motion scope improved significantly. There were no apparent nonspecific inflammatory or rejection reactions. No obvious lacuna was detected between grafting materials and host bone tissues after one year follow-up. The new bone formed in the residual lumen gradually crawled to the center and connected platelike bone tissues, which means that the new bone formed close connections between grafting bone materials and host bone (Figures 2 and 3 ).

Nanocomposite bone material has 300 700 $\mu \mathrm{m}$ macropores and $0.5 \sim 50 \mu \mathrm{m}$ micropores, a maximum porosity ratio of $85 \%$, and a maximum hole rate of $60 \%$. The threedimensional spatial structure composed by macropores and micropores increases the internal surface area, which is beneficial to adsorb proteins, thus improving the activity of alkaline phosphatase (ALP), facilitating bone biomineralization, promoting the process of cell adhesion, growth, proliferation, differentiation, promoting calcium, and phosphorus sediment deposits on the surface, and accelerating osteogenesis. Higher porosity is also advantageous for the transmission of oxygen and nutrients and for the growth of blood vessels and nerves. Thus, good conditions were provided for new bone formation. At the same time, the compressive strength of $\mathrm{n}$ $\mathrm{HA}$ lies in the range of 2.0 10.0 MPa and the content of n-HA in composites was controlled to approximately $65 \%$, which gives it a similar mechanical strength, bending strength, and elastic modulus to cancellous bone. The accumulation of mechanical strength by a porous material structure with the characteristics mentioned above satisfied the requirements to repair bone defects and the demands of early functional exercise after surgery $[12,14,15,24]$. When a lesion involves the bone cross-sectional area $<50 \%$, the sick femur underwent nanocomposite bone material grafting and achieved satisfactorily bone fusion one year after surgery (Figure 4). Yang et al. [23] suggested that the porous materials could improve mechanical strength by providing bone remodeling with mechanical support. Additionally, nanocomposite bone material can effectively avoid the joint collapse of the bonegrafting area or fractures. In our study, 3 patient lesions involved a bone cross-sectional area $>50 \%$ and a diameter of cortical bone defect over $5 \mathrm{~cm}$; therefore, an allogeneic cortex bone plate was tightly embedded in the window of the lesion cortex bone and the femur reconstruction nail was fixed at the same time. Because of the severe lesions, the residual cortical bone is weakened after osteotomy and intralesion curettage, which can cause a large segment of cortical bone defects. Under such conditions, we use allogeneic bone plate grafting to provide longitudinal support and to obtain dispersion axial stress with fixation of femoral reconstruction nails. This can provide a relatively closed frame structure for composite bone grafting and avoid artificial heterotopic ossification in the surrounding soft tissue. In the follow-up period, no nonspecific inflammation, rejection, recurrence, pathologic fractures, or loosened internal fixation was encountered in the 3 patients. The defects were fully occupied by composite bone within a year and a radiographically observable bony union. Meanwhile, there was no significant difference in $\mathrm{G} / \mathrm{N}$ ratio between allograft users and nanocomposite bone material isolate users. In the last follow-up, the users all had satisfactory limb function.

In early reports, preliminary judgment of postoperative bone healing primarily relied on X-ray. Zhang et al. [15] used $\mathrm{X}$-rays and CT scans to make qualitative evaluations about bone union/disunion and the space between grafted bone and host bone tissues. Xiong et al. [14] estimated the extent of bone union through a postoperative osteoplastic ratio (usually presented as a percentage). They used SIEMENS digital imaging software program to measure the X-ray radiation resistance ratio of normal bone around the grafting zone and nonlesion zone for quantitative analysis and then calculated the $\mathrm{G} / \mathrm{N}$ ratio (grafting zone/nonlesion zone). In our study, image data, Harris score, and MSTS score were applied to estimate bone union/disunion and function. X-ray and CT scan showed that $25 \%$ of grafting zones were replaced by new bones in 2 to 3 months after surgery and 50\% in 6 to 9 months after surgery. Bone defects were completely replaced by new bone at one year after surgery, and the mineral density of the bone-grafting areas was closer to normal areas. The mean radiopaque density ratio was $0.78 \pm 0.09$ one year after operation. Hence, we can quantitatively monitor the condition of bone healed and guide the orthopedists to make proper medical judgments on the basis of the G/N ratio. Meanwhile, the functional scores mentioned above sufficiently showed satisfactory function outcomes. After the lesion heals well, the internal fixation is not considered for removal.

Limitations of the Current Study. It should be noted that this study has included 3 cases that underwent allograft cortex bone plate grafting at the same time. Allografts may impact the bone fusion of nanocomposite bone material. Additionally, this study did not undertake a long term followup period, and we need more time to monitor whether there was lesion recurrence, metastasis, or complications caused by the material. In addition, the correcting of deformities, lesion curettage, and solid internal fixation was important to guarantee bone grafting fusion and these factors may affect the results. 


\section{Conclusions}

Nanocomposite bone material has biological safety and good biocompatibility. The material can achieve self-repairing through bone conduction and osteogenic induction and is an efficient bone-grafting material that presents satisfactory effects in the repair of large proximal femoral defects that are due to fibrous dysplasia and should be generalized in the orthopedic field.

\section{Conflict of Interests}

The authors declare that they have no conflict of interests.

\section{Authors' Contribution}

Yun Lang and Ze-ping Yu participated in the collection of clinical data, performed patient follow-ups, and drafted the paper. Yan Xiong made substantial contributions to conception and design of this research and has reviewed the paper for important intellectual content and given final approval of the version to be published. Hong Li and Yong-gang Yan provided the data of the composites' in vitro and animal experiments. Fang Yuan analysed the image data. Hong Duan and Chong-qi Tu were responsible for these operations and participated in project coordination and assisted with the paper. Each author has participated sufficiently in this work to take public responsibility for the appropriate portions of the paper. All authors read and approved the final paper. Yun Lang and Ze-ping Yu contributed equally to this work and should be considered cofirst authors.

\section{Acknowledgment}

The result described in this paper was supported by the National Science and Technology Support Program of the People's Republic of China (2007BAE131304).

\section{References}

[1] A. C. Looker, B. Dawson-Hughes, A. N. A. Tosteson, H. Johansson, J. A. Kanis, and L. J. Melton III, "Hip fracture risk in older US adults by treatment eligibility status based on new National Osteoporosis Foundation guidance," Osteoporosis International, vol. 22, no. 2, pp. 541-549, 2011.

[2] W. R. Moore, S. E. Graves, and G. I. Bain, "Synthetic bone graft substitutes," ANZ Journal of Surgery, vol. 71, no. 6, pp. 354-361, 2001.

[3] A. Schorr, W. Campbell, and M. Schenk, Communication Research and Media Science in Europe: Perspectives for Research and Academic Training in Europe's Changing Media Reality, Mouton de Gruyter, Berlin, Germany, 2003.

[4] A. S. Greenwald, S. D. Boden, V. M. Goldberg, Y. Khan, C. T. Laurencin, and R. N. Rosier, "Bone-graft substitutes: facts, fictions, and applications," Journal of Bone and Joint Surgery A, vol. 83, supplement 2, pp. 98-103, 2001.

[5] L. Lichtenstein, "Polyostotic fibrous dysplasia," Archives of Surgery, vol. 36, no. 5, pp. 874-898, 1938.

[6] E. Ippolito, E. W. Bray, A. Corsi et al., "Natural history and treatment of fibrous dysplasia of bone: a multicenter clinicopathologic study promoted by the European Pediatric Orthopaedic Society," Journal of Pediatric Orthopaedics, Part B, vol. 12, no. 3, pp. 155-177, 2003.

[7] M. Riminucci, I. Saggio, P. G. Robey, and P. Bianco, "Fibrous dysplasia as a stem cell disease," Journal of Bone and Mineral Research, vol. 21, supplement 2, pp. 125-131, 2006.

[8] W. F. Enneking and P. F. Gearen, "Fibrous dysplasia of the femoral neck. Treatment by cortical bone-grafting," The Journal of Bone \& Joint Surgery-American Volume, vol. 68, no. 9, pp. 1415-1422, 1986.

[9] L. Yang, Y. Jing, D. Hong, and T. Chong-Qi, "Valgus osteotomy combined with intramedullary nail for Shepherd's crook deformity in fibrous dysplasia: 14 femurs with a minimum of 4 years follow-up," Archives of Orthopaedic and Trauma Surgery, vol. 130, no. 4, pp. 497-502, 2010.

[10] J. T. Guille, S. J. Kumar, and G. D. Macewen, "Fibrous dysplasia of the proximal part of the femur: long-term results of curettage and bone-grafting and mechanical realignment," The Journal of Bone \& Joint Surgery - American Volume, vol. 80, no. 5, pp. 648658, 1998.

[11] X. Yang, Q. Chen, L.-M. Liu et al., "Comparison of anterior cervical fusion by titanium mesh cage versus nanohydroxyapatite/polyamide cage following single-level corpectomy," International Orthopaedics, vol. 37, no. 12, pp. 2421-2427, 2013.

[12] H. Li, Y. B. Li, Y. G. Yan, G. Zhou, M. Wang, and L. Cheng, "Preparation and biological safety evaluation of porous n-HA/ PA66 composite," Journal of Biomedical Engineering, vol. 25, no. 5, pp. 1126-1129, 2008.

[13] Y. Zhang, Z.-X. Quan, Z.-H. Zhao et al., "Evaluation of anterior cervical reconstruction with titanium mesh cages versus nanohydroxyapatite/polyamide66 cages after 1- or 2-level corpectomy for multilevel cervical spondylotic myelopathy: a retrospective study of 117 patients," PLoS ONE, vol. 9, no. 5, Article ID e96265, 2014

[14] Y. Xiong, C. Ren, B. Zhang et al., "Analyzing the behavior of a porous nano-hydroxyapatite/polyamide 66 (n-HA/PA66) composite for healing of bone defects," International Journal of Nanomedicine, vol. 9, no. 1, pp. 485-494, 2014.

[15] S.-L. Zhang, Y. Zhou, H. Duan et al., "Repairing bone defect with nano-hydroxyapatite and polyamide 66 composite after giant cell tumor operation," Journal of Sichuan UniversityMedical Science Edition, vol. 43, no. 3, pp. 373-377, 2012.

[16] C.-Y. Meng, H. An, D.-M. Jiang et al., "Repair of bone defect with porous composite of nano-hydroxyapatite and polymide," Chinese Journal of Orthopaedic Trauma, vol. 21, no. 3, pp. 187191, 2005.

[17] H. Wang, Y. Li, Y. Zuo, J. Li, S. Ma, and L. Cheng, "Biocompatibility and osteogenesis of biomimetic nanohydroxyapatite/polyamide composite scaffolds for bone tissue engineering," Biomaterials, vol. 28, no. 22, pp. 3338-3348, 2007.

[18] N. N. Mahomed, D. C. Arndt, B. J. McGrory, and W. H. Harris, "The Harris hip score: comparison of patient self-report with surgeon assessment," The Journal of Arthroplasty, vol. 16, no. 5, pp. 575-580, 2001.

[19] W. F. Enneking, W. Dunham, M. C. Gebhardt, M. Malawar, and D. J. Pritchard, "A system for the functional evaluation of reconstructive procedures after surgical treatment of tumors of the musculoskeletal system," Clinical Orthopaedics and Related Research, vol. 1, no. 286, pp. 241-246, 1993. 
[20] T. Lejman and J. Sulko, "Orthopedic management in children with fibrous dysplasia of bone," Chirurgia Narzadów Ruchu $i$ Ortopedia Polska, vol. 64, no. 3, pp. 303-310, 1999.

[21] R. P. Stanton, E. Ippolito, D. Springfield, L. Lindaman, S. Wientroub, and A. Leet, "The surgical management of fibrous dysplasia of bone," Orphanet Journal of Rare Diseases, vol. 7, supplement 1, article S1, 2012.

[22] L. Wu, Y.-B. Li, W.-H. Yang, L. Zhang, and J.-M. Han, "Qualitative and quantitative comparison of human cortical bone, nano-HA and nano-HA/PA66," Chinese Journal of Functional Material, vol. 36, no. 6, pp. 892-895, 2005.

[23] K. Yang, J. Wei, C.-Y. Wang, and Y. Li, "A study on in vitro and in vivo bioactivity of nano hydroxyapatite/polymer biocomposite," Chinese Science Bulletin, vol. 52, no. 2, pp. 267-271, 2007.

[24] Q. Xu, H. Lu, J. Zhang, G. Lu, Z. Deng, and A. Mo, "Tissue engineering scaffold material of porous nanohydroxyapatite/polyamide 66," International Journal of Nanomedicine, vol. 5, pp. 331-335, 2010.

[25] B. Qiao, J. Li, Q. Zhu et al., "Bone plate composed of a ternary nano-hydroxyapatite/polyamide 66/glass fiber composite: biomechanical properties and biocompatibility," International Journal of Nanomedicine, vol. 9, no. 1, pp. 1423-1432, 2014.

[26] Y. Qu, P. Wang, Y. Man, Y. Li, Y. Zuo, and J. Li, "Preliminary biocompatible evaluation of nano-hydroxyapatite/polyamide 66 composite porous membrane," International Journal of Nanomedicine, vol. 5, no. 1, pp. 429-435, 2010.

[27] T. J. Cypher and J. P. Grossman, "Biological principles of bone graft healing," Journal of Foot and Ankle Surgery, vol. 35, no. 5, pp. 413-417, 1996.

[28] H. J. Mankin, F. J. Hornicek, and K. A. Raskin, "Infection in massive bone allografts," Clinical Orthopaedics and Related Research, vol. 3, no. 432, pp. 210-216, 2005. 

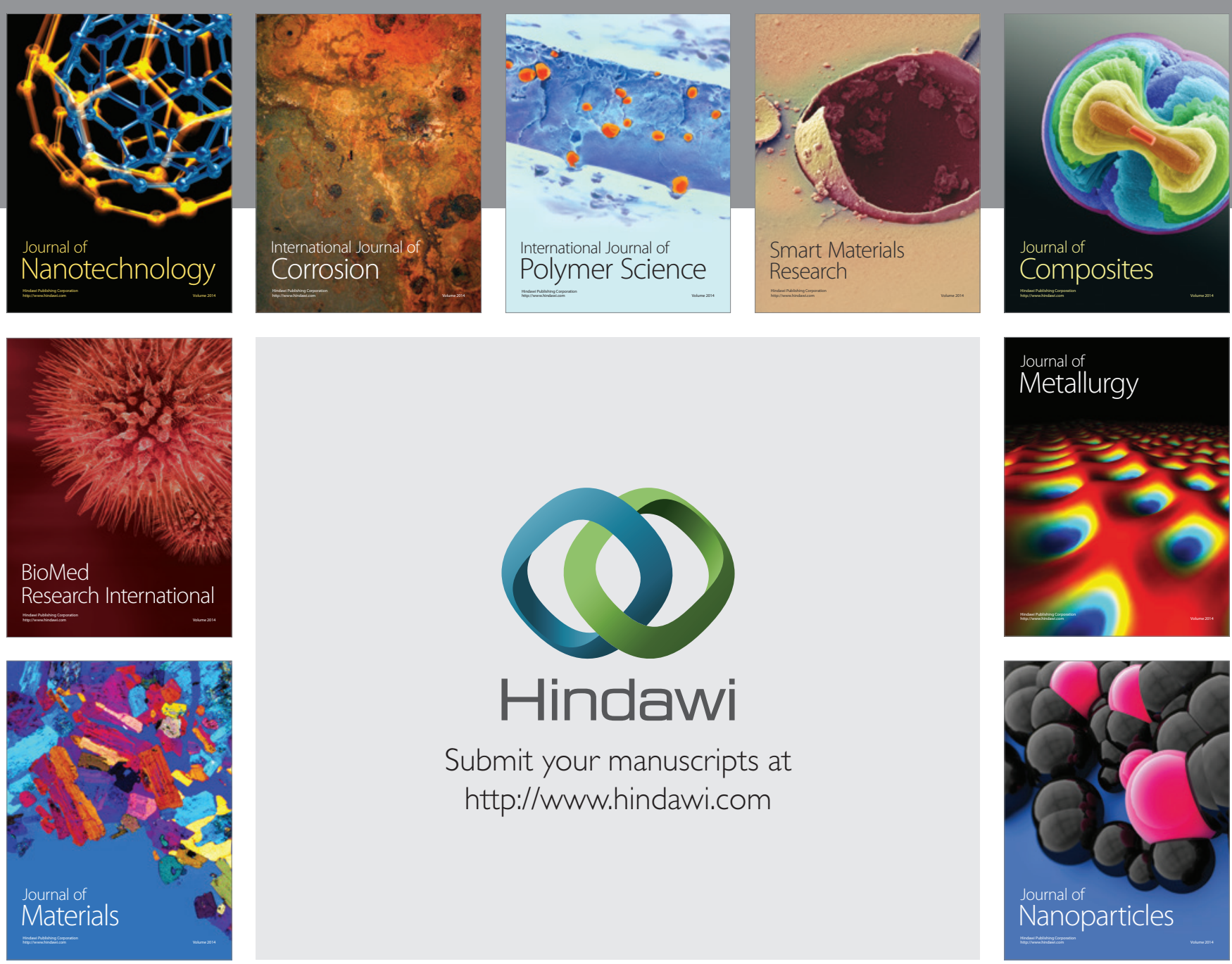

Submit your manuscripts at http://www.hindawi.com
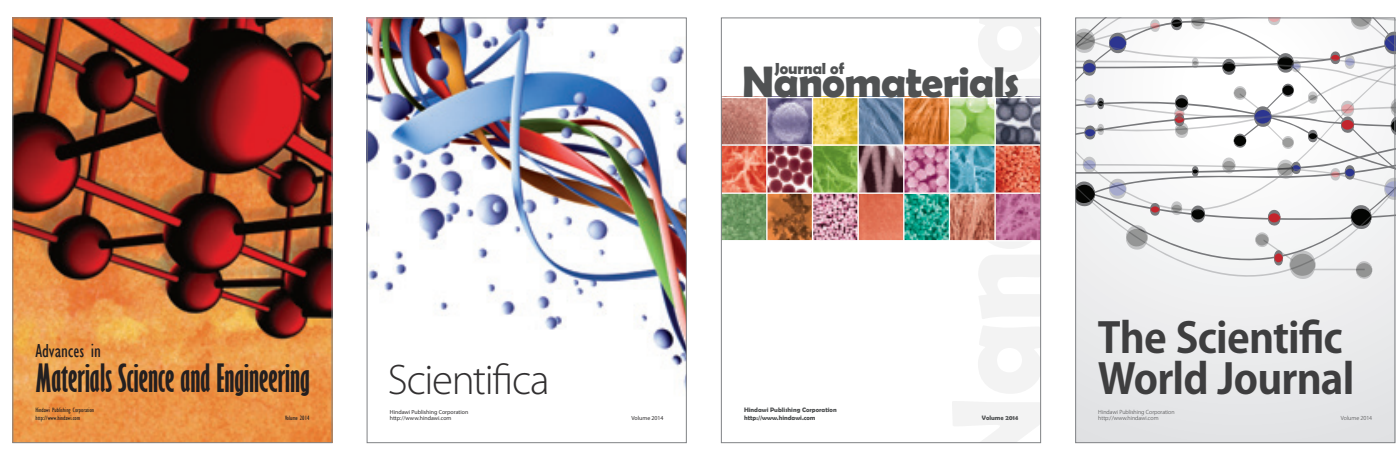

\section{The Scientific World Journal}
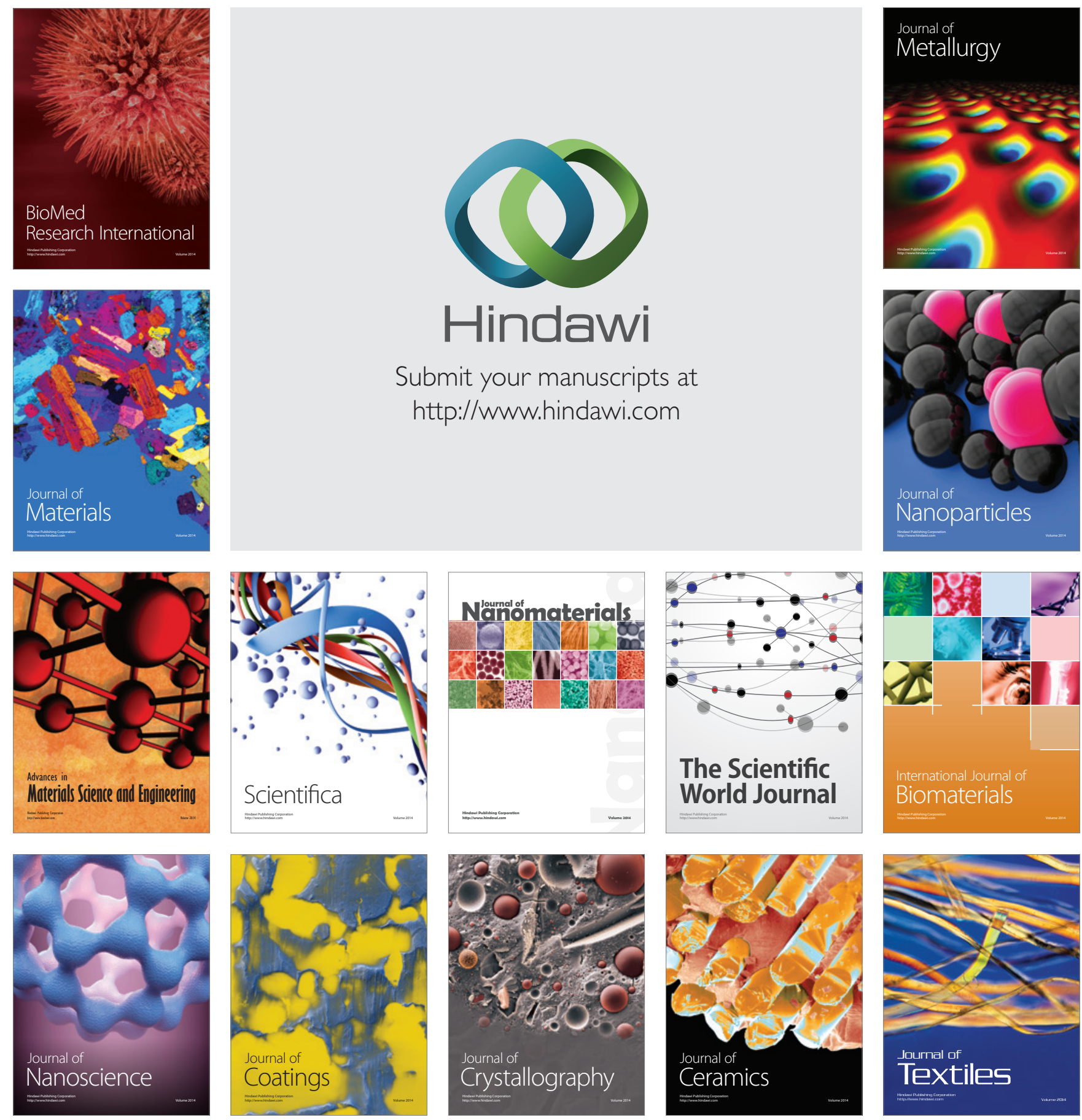